FACTA UNIVERSITATIS (NIŠ)

Ser. Math. Inform. Vol. 35, No 2 (2020), 507-522

https://doi.org/10.22190/FUMI2002507S

\title{
ON STAR COLORING OF DEGREE SPLITTING OF COMB PRODUCT GRAPHS
}

\author{
Ulagammal Subramanian and Vernold Vivin Joseph
}

(C) 2020 by University of Niš, Serbia | Creative Commons Licence: CC BY-NC-ND

\begin{abstract}
A star coloring of a graph $G$ is a proper vertex coloring in which every path on four vertices in $G$ is not bi-colored. The star chromatic number $\chi_{s}(G)$ of $G$ is the least number of colors needed to star color $G$. Let $G=(V, E)$ be a graph with $V=S_{1} \cup S_{2} \cup S_{3} \cup \ldots \cup S_{t} \cup T$ where each $S_{i}$ is a set of all vertices of the same degree with at least two elements and $T=V(G)-\bigcup_{i=1}^{t} S_{i}$. The degree splitting graph $D S(G)$ is obtained by adding vertices $w_{1}, w_{2}, \ldots w_{t}$ and joining $w_{i}$ to each vertex of $S_{i}$ for $1 \leq i \leq t$. The comb product between two graphs $G$ and $H$, denoted by $G \triangleright H$, is a graph obtained by taking one copy of $G$ and $|V(G)|$ copies of $H$ and grafting the $i^{t h}$ copy of $H$ at the vertex $o$ to the $i^{\text {th }}$ vertex of $G$. In this paper, we give the exact value of star chromatic number of degree splitting of comb product of complete graph with complete graph, complete graph with path, complete graph with cycle, complete graph with star graph, cycle with complete graph, path with complete graph and cycle with path graph.
\end{abstract}

Keywords: Star coloring; degree splitting graph; comb product

\section{Introduction}

All graphs in this paper are finite, simple, connected and undirected graph in $[4,5,10]$. The concept of star chromatic number was introduced by Branko Grunbaum in 1973. A star coloring [1, 8, 9] of a graph $G$ is a proper vertex coloring in which every path on four vertices uses at least three distinct colors. Equivalently, in a star coloring, the induced subgraph formed by the vertices of any two colors has connected components that are star graph. The star chromatic number $\chi_{s}(G)$ of $G$ is the least number of colors needed to star color $G$.

Guillaume Fertin et al. [8] determined the star chromatic number of trees, cycles, complete bipartite graphs, outer planar graphs and 2-dimensional grids. They also

Received October 25, 2018; accepted May 19, 2019

2010 Mathematics Subject Classification. Primary 05C15 ; Secondary 05C75 
investigated and gave bounds for the star chromatic number of other families of graphs, such as planar graphs, hypercubes, graphs with bounded treewidth and cubic graphs and planar graphs with high - girth.

Albertson et al. [1] showed that it is NP-complete to determine whether $\chi_{s}(G) \leq$ 3 , even when $G$ is a graph that is both planar and bipartite. Coleman et al. [6] proved that star coloring remains NP-hard problem even on bipartite graphs.

For a given graph $G=(V(G), E(G))$ with $V(G)=S_{1} \cup S_{2} \cup S_{3} \cup \ldots S_{t} \cup T$ where each $S_{i}$ is a set of all vertices of the same degree with at least two elements and $T=V(G)-\bigcup_{i=1}^{t} S_{i}$. The degree splitting graph [11, 12] of $G$, denoted by $D S(G)$, is obtained by adding vertices $w_{1}, w_{2}, \ldots w_{t}$ and joining $w_{i}$ to each vertex of $S_{i}$ for $1 \leq i \leq t$.

Comb product is also same as the hierarchical product graphs was first introduced by Barriére et al. [3] in 2009. Also, the exact value of metric dimension of hierarchical product graphs was obtained by Tavakoli et al. in [14]. Let $G$ and $H$ be two connected graphs. Let $o$ be a vertex of $H$. The comb product between $G$ and $H$, denoted by $G \triangleright H$, is a graph obtained by taking one copy of $G$ and $|V(G)|$ copies of $H$ and grafting the $i^{t h}$ copy of $H$ at the vertex $o$ to the $i^{t h}$ vertex of $G$. By the definition of comb product, we can say that $V(G \triangleright H)=\{(a, u) \mid a \in V(G), u \in V(H)\}$ and $(a, u)(b, v) \in E(G \triangleright H)$ whenever $a=b$ and $u v \in E(H)$, or $a b \in E(G)$ and $u=v=o$. Ridho Alfarisi et al. [2] determined the partition dimension of comb product of path and complete graph and in [7] they also determined the star partition dimension of comb product of cycle and complete graph. Saputro et al. showed the metric dimension of comb product of the connected graphs $G$ and $H$ in [13]. 


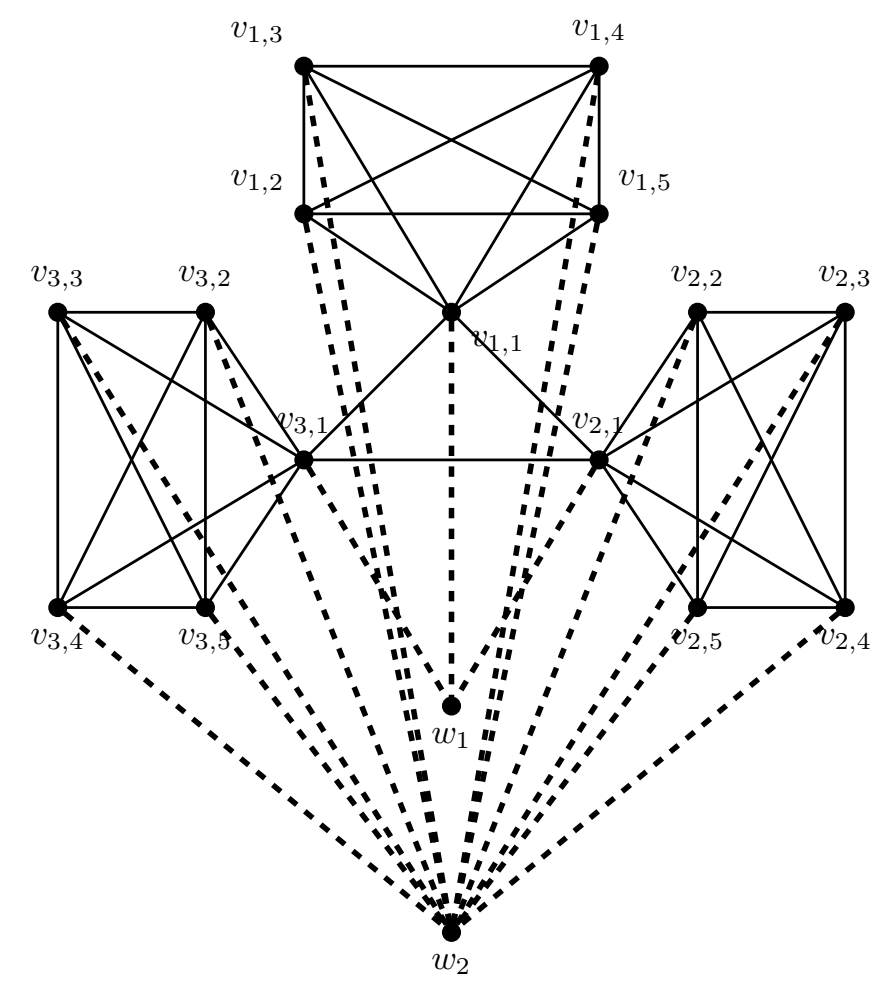

Figure 1: $D S\left(K_{3} \triangleright K_{5}\right)$

In this paper, we have given the exact value of star chromatic number of degree splitting graph of comb product of complete graph with complete graph, complete graph with path, complete graph with cycle, complete graph with star graph, cycle with complete graph, path with complete graph and cycle with path graph denoted by $D S\left(K_{m} \triangleright K_{n}\right), D S\left(K_{m} \triangleright P_{n}\right), D S\left(K_{m} \triangleright C_{n}\right), D S\left(K_{m} \triangleright K_{1, n}\right), D S\left(C_{m} \triangleright K_{n}\right)$, $D S\left(P_{m} \triangleright K_{n}\right)$ and $D S\left(C_{m} \triangleright P_{n}\right)$ respectively.

In order to prove our results, we shall make use of the following theorem by Guillaume et al. [8].

Theorem 1.1. [8] If $C_{n}$ is a cycle with $n \geq 3$ vertices, then

$$
\chi_{s}\left(C_{n}\right)= \begin{cases}4, & \text { when } \quad n=5 \\ 3, & \text { otherwise. }\end{cases}
$$

Proof. The proof of the theorem can be found in [8]. 


\section{Main Results}

In the following subsections, we will find the star chromatic number of degree splitting graph of comb product of complete with complete graph, complete with path, complete with cycle, complete with star graph, comb product of cycle with complete, path with complete and cycle with path graph denoted by $D S\left(K_{m} \triangleright K_{n}\right)$, $D S\left(K_{m} \triangleright C_{n}\right), D S\left(K_{m} \triangleright K_{1, n}\right), D S\left(K_{m} \triangleright P_{n}\right), D S\left(C_{m} \triangleright K_{n}\right), D S\left(P_{m} \triangleright K_{n}\right)$ and $D S\left(C_{m} \triangleright P_{n}\right)$ respectively. Figure 1 shows an example of degree splitting of comb product $\left(K_{3} \triangleright K_{5}\right)$.

\subsection{Star Coloring of Degree Splitting of $\left(K_{m} \triangleright K_{n}\right)$}

The comb product between $K_{m}$ and $K_{n}$, denoted by $K_{m} \triangleright K_{n}$ has vertex set

$$
V\left(K_{m} \triangleright K_{n}\right)=\left\{v_{i, j}: 1 \leq i \leq m, 1 \leq j \leq n\right\}
$$

and edge set

$$
\begin{aligned}
E\left(K_{m} \triangleright K_{n}\right)= & \left\{v_{i, 1} v_{i+k, 1}: 1 \leq i \leq m, 1 \leq k \leq m-i\right\} \\
& \bigcup\left\{v_{i, j} v_{i, j+k}: 1 \leq i \leq m, 1 \leq j \leq n, 1 \leq k \leq n-j\right\} .
\end{aligned}
$$

Thus

$$
\left|V\left(K_{m} \triangleright K_{n}\right)\right|=m n
$$

and

$$
\left|E\left(K_{m} \triangleright K_{n}\right)\right|=\frac{m n(n-1)+m(m-1)}{2} .
$$

Theorem 2.1. Let $K_{m}$ and $K_{n}$ be two complete graphs of order $m, n \geq 3$ and $m \leq n$, then

$$
\chi_{s}\left(D S\left(K_{m} \triangleright K_{n}\right)\right)=m+n .
$$

Proof. We have,

$$
V\left(K_{m} \triangleright K_{n}\right)=\left\{v_{i, j}: 1 \leq i \leq m, 1 \leq j \leq n\right\}=S_{1} \cup S_{2}
$$

where

$$
S_{1}=\left\{v_{i, 1}: 1 \leq i \leq m\right\}
$$

and

$$
S_{2}=\left\{v_{i, j}: 1 \leq i \leq m, 2 \leq j \leq n\right\} .
$$

To obtain $D S\left(K_{m} \triangleright K_{n}\right)$ from $K_{m} \triangleright K_{n}$, we add two vertices $w_{1}$ and $w_{2}$ corresponding to $S_{1}$ and $S_{2}$ respectively. Thus, we get $V\left(D S\left(K_{m} \triangleright K_{n}\right)\right)=V\left(K_{m} \triangleright K_{n}\right) \cup$ $\left\{w_{1}, w_{2}\right\}$. First we find the upper bound for $\chi_{s}\left(D S\left(K_{m} \triangleright K_{n}\right)\right)$.

Clearly, $m+n$ colors are needed at least to star color $D S\left(K_{m} \triangleright K_{n}\right)$. We now distinguish $n$ as three cases: For every $1 \leq i \leq m$, 
Case(i): When $n \equiv 3(\bmod 3)$.

$$
\begin{gathered}
\sigma\left(v_{i, 3 k-2}\right)=i+j-1, \text { for } 1 \leq k \leq \frac{n}{3} \\
\sigma\left(v_{i, 3 k-1}\right)=i+j-1, \text { for } 1 \leq k \leq \frac{n}{3} \\
\sigma\left(v_{i, 3 k}\right)=i+j-1, \text { for } 1 \leq k \leq \frac{n}{3}
\end{gathered}
$$

and

$$
\sigma\left(w_{1}\right)=\sigma\left(w_{2}\right)=m+n
$$

Case(ii): When $n \equiv 1(\bmod 3)$.

$$
\begin{gathered}
\sigma\left(v_{i, 3 k-2}\right)=i+j-1, \text { for } 1 \leq k \leq\left\lceil\frac{n}{3}\right\rceil \\
\sigma\left(v_{i, 3 k-1}\right)=i+j-1, \text { for } 1 \leq k \leq\left\lfloor\frac{n}{3}\right\rfloor \\
\sigma\left(v_{i, 3 k}\right)=i+j-1, \text { for } 1 \leq k \leq\left\lfloor\frac{n}{3}\right\rfloor
\end{gathered}
$$

and

$$
\sigma\left(w_{1}\right)=\sigma\left(w_{2}\right)=m+n
$$

Case(iii): When $n \equiv 2(\bmod 3)$.

$$
\begin{gathered}
\sigma\left(v_{i, 3 k-2}\right)=i+j-1, \text { for } 1 \leq k \leq\left\lceil\frac{n}{3}\right\rceil \\
\sigma\left(v_{i, 3 k-1}\right)=i+j-1, \text { for } 1 \leq k \leq\left\lceil\frac{n}{3}\right\rceil \\
\sigma\left(v_{i, 3 k}\right)=i+j-1, \text { for } 1 \leq k \leq\left\lfloor\frac{n}{3}\right\rfloor
\end{gathered}
$$

and

$$
\sigma\left(w_{1}\right)=\sigma\left(w_{2}\right)=m+n .
$$

Thus, the upper bound for the star coloring of $\left(D S\left(K_{m} \triangleright K_{n}\right)\right) \leq m+n$.

Now, we prove the lower bound for $\chi_{s}\left(D S\left(K_{m} \triangleright K_{n}\right)\right)$.

Suppose $\chi_{s}\left(D S\left(K_{m} \triangleright K_{n}\right)\right)<m+n$. Let $\chi_{s}\left(D S\left(K_{m} \triangleright K_{n}\right)\right)=m+n-1$, then there exists a bicolored path $P_{4}$. Since $\left\{v_{1, i}\right\}$ induce a clique of order $n$ (say $K_{n}$ ). If we assign the same $n$ colors to the second copy of $K_{n}$, then we get a path on four vertices between these clique which is bicolored, a contradiction for proper star coloring. Thus, $\chi_{s}\left(D S\left(K_{m} \triangleright K_{n}\right)\right)=m+n-1$ color is impossible. Therefore, the lower bound of $\chi_{s}\left(D S\left(K_{m} \triangleright K_{n}\right)\right) \geq m+n$. Thus we get the lower and upper bound of $\chi_{s}\left(D S\left(K_{m} \triangleright K_{n}\right)\right)$. Hence $\chi_{s}\left(D S\left(K_{m} \triangleright K_{n}\right)\right)=m+n$. This completes the proof of the theorem. 


\subsection{Star Coloring of Degree Splitting of $\left(K_{m} \triangleright C_{n}\right)$}

A graph $K_{m} \triangleright C_{n}$ has vertex set

$$
V\left(K_{m} \triangleright C_{n}\right)=\left\{v_{i, j}: 1 \leq i \leq m, 1 \leq j \leq n\right\}
$$

and edge set

$$
\begin{aligned}
\left|E\left(K_{m} \triangleright C_{n}\right)\right|= & \left\{v_{i, 1} v_{i+k, 1}: 1 \leq i \leq m, 1 \leq k \leq m-i\right\} \\
& \bigcup\left\{v_{i, j} v_{i, j+1}: 1 \leq i \leq m-1,1 \leq j \leq n-1\right\} \bigcup\left\{v_{m, 1} v_{1,1}\right\} .
\end{aligned}
$$

Thus

$$
\left|V\left(K_{m} \triangleright C_{n}\right)\right|=m n
$$

and

$$
\left|E\left(K_{m} \triangleright C_{n}\right)\right|=\frac{m(m-1)+2 m n}{2} .
$$

Theorem 2.2. Let $K_{m}$ and $C_{n}$ be two connected graphs of order $m \geq 4$ and $n \geq 5$, then

$$
\chi_{s}\left(D S\left(K_{m} \triangleright C_{n}\right)\right)=m+1 .
$$

Proof. We have

$$
V\left(K_{m} \triangleright C_{n}\right)=\left\{v_{i, j}: 1 \leq i \leq m, 1 \leq j \leq n\right\}=S_{1} \cup S_{2}
$$

where

$$
S_{1}=\left\{v_{i, 1}: 1 \leq i \leq m\right\}
$$

and

$$
S_{2}=\left\{v_{i, j}: 1 \leq i \leq m, 2 \leq j \leq n\right\} .
$$

To obtain $D S\left(K_{m} \triangleright C_{n}\right)$ from $K_{m} \triangleright C_{n}$, we add two vertices $w_{1}$ and $w_{2}$ corresponding to $S_{1}$ and $S_{2}$ respectively. Thus we get

$$
V\left(D S\left(K_{m} \triangleright C_{n}\right)\right)=\left\{v_{i, j}: 1 \leq i \leq m, 1 \leq j \leq n\right\} \bigcup\left\{w_{1}, w_{2}\right\} .
$$

We first prove the lower bound for the star chromatic number of degree splitting of comb product of complete graph with cycle. For this, we show that any coloring with $m$ colors will give us at least one bicolored path of length 4 . Since each $\left\{v_{i, 1}: 1 \leq i \leq m\right\}$ is adjacent to $w_{1}$, it gives a complete graph of order $m+1$. Thus, no coloring that uses $m$ colors can be a star coloring. Therefore, the lower bound of star chromatic number is $\chi_{s}\left(D S\left(K_{m} \triangleright C_{n}\right)\right) \geq m+1$.

Now, we prove the upper bound for the star chromatic number of degree splitting of $\left(K_{m} \triangleright C_{n}\right)$. Since the complete graph has the chromatic number $m$. We assign the $m$ colors to the $m n$ vertices of the graph $K_{m} \triangleright C_{n}$ alternatively and we assign $\sigma\left(w_{1}\right)=\sigma\left(w_{2}\right)=m+1$. Thus the upper bound of the $\chi_{s}\left(D S\left(K_{m} \triangleright C_{n}\right)\right) \leq m+1$.

Thus we get the lower and upper bound of the $\chi_{s}\left(D S\left(K_{m} \triangleright C_{n}\right)\right)$. Therefore,

$$
\chi_{s}\left(D S\left(K_{m} \triangleright C_{n}\right)\right)=m+1 .
$$

This concludes the proof of the theorem. 


\subsection{Star Coloring of Degree Splitting of $\left(K_{m} \triangleright P_{n}\right)$}

A graph $K_{m} \triangleright P_{n}$ has vertex set

$$
V\left(K_{m} \triangleright P_{n}\right)=\left\{v_{i, j}: 1 \leq i \leq m, 1 \leq j \leq n\right\}
$$

and edge set

$$
\begin{aligned}
E\left(K_{m} \triangleright P_{n}\right)= & \left\{v_{i, 1} v_{i+k, 1}: 1 \leq i \leq m, 1 \leq k \leq m-i\right\} \\
& \bigcup\left\{v_{i, j} v_{i, j+1}: 1 \leq i \leq m, 1 \leq j \leq n-1\right\} .
\end{aligned}
$$

Thus

$$
\left|V\left(K_{m} \triangleright P_{n}\right)\right|=m n
$$

and

$$
\left|E\left(K_{m} \triangleright P_{n}\right)\right|=\frac{m(m-1)+2 m(n-1)}{2} .
$$

Theorem 2.3. Let $K_{m}$ be a complete graph of order $m \geq 3$ and $P_{n}$ be a path graph of order $n \geq 3$ then,

$$
\chi_{s}\left(D S\left(K_{m} \triangleright P_{n}\right)\right)=m+1 .
$$

Proof. We have

$$
V\left(K_{m} \triangleright P_{n}\right)=\left\{v_{i, j}: 1 \leq i \leq m, 1 \leq j \leq n\right\}=S_{1} \cup S_{2} \cup S_{3}
$$

where

$$
\begin{gathered}
S_{1}=\left\{v_{i, 1}: 1 \leq i \leq m\right\} \\
S_{2}=\left\{v_{i, j}: 1 \leq i \leq m, 2 \leq j \leq n-1\right\}
\end{gathered}
$$

and

$$
S_{3}=\left\{v_{i, n}: 1 \leq i \leq m\right\} .
$$

To obtain $D S\left(K_{m} \triangleright P_{n}\right)$ from $K_{m} \triangleright P_{n}$, we add three vertices $w_{1}, w_{2}$ and $w_{3}$ corresponding to $S_{1}, S_{2}$ and $S_{3}$ respectively. Thus, $V\left(D S\left(K_{m} \triangleright P_{n}\right)\right)=V\left(K_{m} \triangleright P_{n}\right) \cup$ $\left\{w_{1}, w_{2}, w_{3}\right\}$. Now, we assign the following coloring pattern:

For every $1 \leq i \leq m$

For $n \equiv 1(\bmod 3)$

$$
\begin{gathered}
\sigma\left(v_{i, 3 k-2}\right)=i+j-1(\bmod m), \text { for } 1 \leq k \leq\left\lceil\frac{n}{3}\right\rceil \\
\sigma\left(v_{i, 3 k-1}\right)=i+j-1(\bmod m), \text { for } 1 \leq k \leq\left\lfloor\frac{n}{3}\right\rfloor \\
\sigma\left(v_{i, 3 k}\right)=i+j-1(\bmod m), \text { for } 1 \leq k \leq\left\lfloor\frac{n}{3}\right\rfloor
\end{gathered}
$$


and

$$
\sigma\left(w_{1}\right)=\sigma\left(w_{2}\right)=\sigma\left(w_{3}\right)=m+1
$$

For $n \equiv 2(\bmod 3)$

$$
\begin{gathered}
\sigma\left(v_{i, 3 k-2}\right)=i+j-1(\bmod m), \text { for } 1 \leq k \leq\left\lceil\frac{n}{3}\right\rceil \\
\sigma\left(v_{i, 3 k-1}\right)=i+j-1(\bmod m), \text { for } 1 \leq k \leq\left\lceil\frac{n}{3}\right\rceil \\
\sigma\left(v_{i, 3 k}\right)=i+j-1(\bmod m), \text { for } 1 \leq k \leq\left\lfloor\frac{n}{3}\right\rfloor
\end{gathered}
$$

and

$$
\sigma\left(w_{1}\right)=\sigma\left(w_{2}\right)=\sigma\left(w_{3}\right)=m+1
$$

For $n \equiv 3(\bmod 3)$

$$
\begin{gathered}
\sigma\left(v_{i, 3 k-2}\right)=i+j-1(\bmod m), \text { for } 1 \leq k \leq \frac{n}{3} \\
\sigma\left(v_{i, 3 k-1}\right)=i+j-1(\bmod m), \text { for } 1 \leq k \leq \frac{n}{3} \\
\sigma\left(v_{i, 3 k}\right)=i+j-1(\bmod m), \text { for } 1 \leq k \leq \frac{n}{3}
\end{gathered}
$$

and

$$
\sigma\left(w_{1}\right)=\sigma\left(w_{2}\right)=\sigma\left(w_{3}\right)=m+1 .
$$

Thus the upper bound of star coloring of degree splitting of $\left(K_{m} \triangleright P_{n}\right) \leq m+1$.

Now, we prove the lower bound of $\chi_{s}\left(D S\left(K_{m} \triangleright P_{n}\right)\right)$. Suppose the lower bound of the

$$
\chi_{s}\left(D S\left(K_{m} \triangleright P_{n}\right)\right)<m+1 .
$$

That is

$$
\chi_{s}\left(D S\left(K_{m} \triangleright P_{n}\right)\right)=m .
$$

We must assign $m$ colors for $\left\{v_{i, 1}, 1 \leq i \leq m\right\}$ for proper star coloring. Since each $\left\{v_{i, 1}\right\}$ is adjacent to $w_{1}$, it gives a complete graph of order $m+1$. Therefore $\chi_{s}\left(D S\left(K_{m} \triangleright P_{n}\right)\right)$ with $m$ colors is impossible. Therefore $\chi_{s}\left(D S\left(K_{m} \triangleright P_{n}\right)\right) \geq$ $m+1$. Hence, $\chi_{s}\left(\left(D S\left(K_{m} \triangleright P_{n}\right)\right)=m+1\right.$. This concludes the proof of the theorem. 


\subsection{Star Coloring of Degree Splitting of $\left(K_{m} \triangleright K_{1, n}\right)$}

A graph $K_{m} \triangleright K_{1, n}$ has a vertex set

$$
V\left(K_{m} \triangleright K_{1, n}\right)=\left\{v_{i, j}: 1 \leq i \leq m, 1 \leq j \leq n\right\}
$$

and edge set

$$
\begin{aligned}
E\left(K_{m} \triangleright K_{1, n}\right)= & \left\{v_{i, 1} v_{i+k, 1}: 1 \leq i \leq m-1,1 \leq k \leq m-i\right\} \\
& \bigcup\left\{v_{i, 1} v_{i, j+1}: 1 \leq i \leq m, 1 \leq j \leq n\right\} .
\end{aligned}
$$

Thus

$$
\left|V\left(K_{m} \triangleright K_{1, n}\right)\right|=m n
$$

and

$$
\left|E\left(K_{m} \triangleright K_{1, n}\right)\right|=\frac{m(m-1)+2 m n}{2} .
$$

Theorem 2.4. Let $K_{m}$ be a complete graph of order $m,(m \geq 3)$ and $K_{1, n}$ be a star graph with $n+1$ vertices $(n \geq 2)$ then

$$
\chi_{s}\left(D S\left(K_{m} \triangleright K_{1, n}\right)\right)=m+1 .
$$

Proof. We have

$$
V\left(K_{m} \triangleright K_{1, n}\right)=\left\{v_{i, j}: 1 \leq i \leq m, 1 \leq j \leq n\right\}=S_{1} \cup S_{2} .
$$

To obtain $D S\left(K_{m} \triangleright K_{1, n}\right)$ from $\left(K_{m} \triangleright K_{1, n}\right)$, we add two vertices $w_{1}$ and $w_{2}$ corresponding to $S_{1}$ and $S_{2}$ respectively, where

$$
S_{1}=\left\{v_{i, 1}: 1 \leq i \leq m\right\}
$$

and

$$
S_{2}=\left\{v_{i, j}: 1 \leq i \leq m, 2 \leq j \leq n\right\}
$$

Thus we get

$$
V\left(D S\left(K_{m} \triangleright K_{1, n}\right)\right)=V\left(K_{m} \triangleright K_{1, n}\right) \cup\left\{w_{1}, w_{2}\right\} .
$$

Now we assign the coloring pattern as follows:

For every $1 \leq i \leq m$, assign $i$ to $\sigma\left(v_{i, 1}\right)$, and

For $2 \leq j \leq n$ assign

$$
\sigma\left(v_{i, j}\right)=\left\{\begin{array}{l}
2 \text { if } i \equiv 1(\bmod 3) \\
3 \text { if } i \equiv 2(\bmod 3) \\
1 \text { if } i \equiv 3(\bmod 3)
\end{array}\right.
$$

alternatively 
and

$$
\sigma\left(w_{1}\right)=\sigma\left(w_{2}\right)=m+1 .
$$

Thus $\chi_{s}\left(\left(D S\left(K_{m} \triangleright K_{1, n}\right)\right) \geq m+1\right.$.

Now, we prove the lower bound of $\chi_{s}\left(D S\left(K_{m} \triangleright K_{1, n}\right)\right)$. Suppose the lower bound of the $\chi_{s}\left(D S\left(K_{m} \triangleright K_{1, n}\right)\right)<m+1$. That is $\chi_{s}\left(D S\left(K_{m} \triangleright K_{1, n}\right)\right)=m$. We must assign $m$ colors for $\left\{v_{i, 1}: 1 \leq i \leq m\right\}$ for proper star coloring. Since each $\left\{v_{i, 1}\right\}$ is adjacent to $w_{1}$, it gives a complete graph of order $m+1$. Therefore $\chi_{s}\left(D S\left(K_{m} \triangleright K_{1, n}\right)\right)$ with $m$ color is impossible. Therefore $\chi_{s}\left(D S\left(K_{m} \triangleright K_{1, n}\right)\right) \geq$ $m+1$. Thus we get the lower and upper bound of $\chi_{s}\left(D S\left(K_{m} \triangleright K_{1, n}\right)\right)$. Hence, $\chi_{s}\left(D S\left(K_{m} \triangleright K_{1, n}\right)\right)=m+1$. It concludes the proof of the theorem.

\subsection{Star Coloring of Degree Splitting of $\left(C_{m} \triangleright K_{n}\right)$}

A graph $C_{m} \triangleright K_{n}$ has vertex set

$$
V\left(C_{m} \triangleright K_{n}\right)=\left\{v_{i, j}: 1 \leq i \leq m, 1 \leq j \leq n\right\}
$$

and edge set

$$
\begin{aligned}
E\left(C_{m} \triangleright K_{n}\right)= & \left\{v_{i, 1} v_{i+1,1}: 1 \leq i \leq m-1\right\} \\
& \bigcup\left\{v_{m, 1} v_{1,1}\right\} \bigcup\left\{v_{i, j} v_{i, j+k}: 1 \leq i \leq m, 1 \leq j \leq n, 1 \leq k \leq n-j\right\} .
\end{aligned}
$$

Thus

$$
\left|V\left(C_{m} \triangleright K_{n}\right)\right|=m n
$$

and

$$
\left|E\left(C_{m} \triangleright K_{n}\right)\right|=\frac{m n^{2}-m n+2 m}{2} .
$$

Theorem 2.5. Let $C_{m}$ and $K_{n}$ be two connected graphs of order $m>n$ and $m>3, n \geq 3$, then

$$
\chi_{s}\left(D S\left(C_{m} \triangleright K_{n}\right)\right)=m+1 .
$$

Proof. We have

$$
V\left(C_{m} \triangleright K_{n}\right)=\left\{v_{i, j}: 1 \leq i \leq m, 1 \leq j \leq n\right\}=S_{1} \cup S_{2}
$$

where

$$
S_{1}=\left\{v_{i, 1}: 1 \leq i \leq m\right\}
$$

and

$$
S_{2}=\left\{v_{i, j}: 1 \leq i \leq m, 2 \leq j \leq n\right\} .
$$

To obtain $D S\left(C_{m} \triangleright K_{n}\right)$ from $C_{m} \triangleright K_{n}$, we add two vertices $w_{1}$ and $w_{2}$ corresponding to $S_{1}$ and $S_{2}$ respectively. Thus we get $V\left(D S\left(C_{m} \triangleright K_{n}\right)\right)=V\left(C_{m} \triangleright K_{n}\right) \cup$ $\left\{w_{1}, w_{2}\right\}$. First we find the upper bound for $\chi_{s}\left(D S\left(C_{m} \triangleright K_{n}\right)\right)$.

We define the coloring pattern as follows: 
For every $1 \leq i \leq m$ and $1 \leq j \leq n$,

$$
\sigma\left(v_{i, j}\right)=i+j-1(\bmod m)
$$

and also

$$
\sigma\left(w_{1}\right)=\sigma\left(w_{2}\right)=m+1 .
$$

Thus the upper bound for star chromatic number of $\left(D S\left(C_{m} \triangleright K_{n}\right)\right) \leq m+1$.

Now, we prove the lower bound for $\chi_{s}\left(\left(D S\left(C_{m} \triangleright K_{n}\right)\right)\right.$.

Suppose the lower bound of $\chi_{s}\left(\left(D S\left(C_{m} \triangleright K_{n}\right)\right)<m+1\right.$. Let $\chi_{s}\left(\left(D S\left(C_{m} \triangleright K_{n}\right)\right)=\right.$ $m$, then there exist a bicolored path $P_{4}$. Since $\left\{v_{1, i}\right\}$ induce a clique of order $n$. If we assign the same $n$ colors to the second copy of the clique, then we get a path on four vertices between these cliques which is bicolored, a contradiction for proper star coloring. Thus we obtain $\chi_{s}\left(\left(D S\left(C_{m} \triangleright K_{n}\right)\right)=m\right.$ color is impossible. It concludes that the lower bound is $\chi_{s}\left(\left(D S\left(C_{m} \triangleright K_{n}\right)\right) \geq m+1\right.$. Therefore, $\chi_{s}\left(\left(D S\left(C_{m} \triangleright K_{n}\right)\right)=m+1\right.$. Hence the proof of the theorem.

\subsection{Star Coloring of Degree Splitting of $\left(P_{m} \triangleright K_{n}\right)$}

A graph $P_{m} \triangleright K_{n}$ has a vertex set

$$
V\left(P_{m} \triangleright K_{n}\right)=\left\{v_{i, j}: 1 \leq i \leq m, 1 \leq j \leq n\right\}
$$

and edge set

$$
\begin{aligned}
E\left(P_{m} \triangleright K_{n}\right)= & \left\{v_{i, 1} v_{i+1,1}: 1 \leq i \leq m-1\right\} \\
& \bigcup\left\{v_{i, j} v_{i, j+k}: 1 \leq i \leq m, 1 \leq j \leq n, 1 \leq k \leq n-j\right\} .
\end{aligned}
$$

Thus

$$
\left|V\left(P_{m} \triangleright K_{n}\right)\right|=m n
$$

and

$$
\left|E\left(P_{m} \triangleright K_{n}\right)\right|=\frac{m n(n-1)+2(m-1)}{2} .
$$

Theorem 2.6. Let $P_{m}$ be a path graph of order $m \geq 4$ and $K_{n}$ be a complete graph with $n \geq 2$, then

$$
\chi_{s}\left(D S\left(P_{m} \triangleright K_{n}\right)\right)=n+2 \text {. }
$$

Proof. We have

$$
V\left(P_{m} \triangleright K_{n}\right)=\left\{v_{i, j}: 1 \leq i \leq m, 2 \leq j \leq n\right\}=S_{1} \cup S_{2} \cup S_{3}
$$

where

$$
\begin{gathered}
S_{1}=\left\{v_{1,1}, v_{m, 1}\right\}, \\
S_{2}=\left\{v_{i, 1}: 2 \leq i \leq m-1\right\}
\end{gathered}
$$


and

$$
S_{3}=\left\{v_{i, j}: 1 \leq i \leq m, 2 \leq j \leq n\right\} .
$$

To obtain $D S\left(P_{m} \triangleright K_{n}\right)$ from $P_{m} \triangleright K_{n}$, we add three vertices $w_{1}, w_{2}$ and $w_{3}$ corresponding to $S_{1}, S_{2}$ and $S_{3}$ respectively. Thus we get $V\left(D S\left(P_{m} \triangleright K_{n}\right)\right)=$ $\left\{v_{i, j}: 1 \leq i \leq m ; 1 \leq j \leq n\right\} \cup\left\{w_{1}, w_{2}, w_{3}\right\}$. First we find the upper bound for $\chi_{s}\left(D S\left(P_{m} \triangleright K_{n}\right)\right)$. For every $1 \leq i \leq m$,

For $n \equiv 1(\bmod 3)$

$$
\begin{gathered}
\sigma\left(v_{i, 3 k-2}\right)=i+j-1(\bmod n+1), \text { for } 1 \leq k \leq\left\lceil\frac{n}{3}\right\rceil \\
\sigma\left(v_{i, 3 k-1}\right)=i+j-1(\bmod n+1), \text { for } 1 \leq k \leq\left\lceil\frac{n}{3}\right\rceil-1 \\
\sigma\left(v_{i, 3 k}\right)=i+j-1(\bmod n+1), \text { for } 1 \leq k \leq\left\lceil\frac{n}{3}\right\rceil-1
\end{gathered}
$$

and

$$
\sigma\left(w_{1}\right)=\sigma\left(w_{2}\right)=\sigma\left(w_{3}\right)=n+2 .
$$

For $n \equiv 2(\bmod 3)$

$$
\begin{gathered}
\sigma\left(v_{i, 3 k-2}\right)=i+j-1(\bmod n+1), \text { for } 1 \leq k \leq\left\lceil\frac{n}{3}\right\rceil \\
\sigma\left(v_{i, 3 k-1}\right)=i+j-1(\bmod n+1), \text { for } 1 \leq k \leq\left\lceil\frac{n}{3}\right\rceil \\
\sigma\left(v_{i, 3 k}\right)=i+j-1(\bmod n+1), \text { for } 1 \leq k \leq\left\lceil\frac{n}{3}\right\rceil-1
\end{gathered}
$$

and

$$
\sigma\left(w_{1}\right)=\sigma\left(w_{2}\right)=\sigma\left(w_{3}\right)=n+2 .
$$

For $n \equiv 3(\bmod 3)$

$$
\begin{gathered}
\sigma\left(v_{i, 3 k-2}\right)=i+j-1(\bmod n+1), \text { for } 1 \leq k \leq \frac{n}{3} \\
\sigma\left(v_{i, 3 k-1}\right)=i+j-1(\bmod n+1), \text { for } 1 \leq k \leq \frac{n}{3} \\
\sigma\left(v_{i, 3 k}\right)=i+j-1(\bmod n+1), \text { for } 1 \leq k \leq \frac{n}{3}
\end{gathered}
$$

and

$$
\sigma\left(w_{1}\right)=\sigma\left(w_{2}\right)=\sigma\left(w_{3}\right)=n+2 .
$$

Thus $\chi_{s}\left(D S\left(P_{m} \triangleright K_{n}\right) \leq n+2\right.$. 
Now, we prove that $\chi_{s}\left(D S\left(P_{m} \triangleright K_{n}\right) \geq n+2\right.$. Suppose $\chi_{s}\left(D S\left(P_{m} \triangleright K_{n}\right)<\right.$ $n+2$. That is $\chi_{s}\left(D S\left(P_{m} \triangleright K_{n}\right)=n+1\right.$. Since $\left\{v_{1, i}\right\}$ induce a clique of order $n$. If we assign the same $n$ colors to the second copy of the clique, then we get a path on four vertices between these cliques which is bicolored, a contradiction for proper star coloring. Thus $\chi_{s}\left(D S\left(P_{m} \triangleright K_{n}\right) \geq n+1\right.$. Therefore, $\chi_{s}\left(D S\left(P_{m} \triangleright K_{n}\right)=n+2\right.$. Hence, there is a another proof to the theorem.

\subsection{Star Coloring of Degree Splitting of $\left(C_{m} \triangleright P_{n}\right)$}

A graph $C_{m} \triangleright P_{n}$ has vertex set

$$
V\left(C_{m} \triangleright P_{n}\right)=\left\{v_{i, j}: 1 \leq i \leq m, 1 \leq j \leq n\right\}
$$

and edge set

$$
\begin{aligned}
E\left(C_{m} \triangleright P_{n}\right)= & \left\{v_{i, 1} v_{i+1,1}: 1 \leq i \leq m-1\right\} \\
& \bigcup\left\{v_{m, 1} v_{1,1}\right\} \bigcup\left\{v_{i, j} v_{i, j+1}: 1 \leq i \leq m, 1 \leq j \leq n-1\right\} .
\end{aligned}
$$

Thus

$$
\left|V\left(C_{m} \triangleright P_{n}\right)\right|=m n
$$

and

$$
\left|E\left(C_{m} \triangleright P_{n}\right)\right|=m+m(n-1) .
$$

Theorem 2.7. Let $C_{m}$ be a cycle of length $m \geq 3$ and $P_{n}$ be a path of length $n \geq 3$ then,

$$
\chi_{s}\left(D S\left(C_{m} \triangleright P_{n}\right)\right)=\left\{\begin{array}{l}
4, \text { if } m=3 k, k \geq 1 \\
5, \text { otherwise }
\end{array} .\right.
$$

Proof. We have

$$
V\left(C_{m} \triangleright P_{n}\right)=\left\{v_{i, j}: 1 \leq i \leq m, 2 \leq j \leq n\right\}=S_{1} \cup S_{2} \cup S_{3}
$$

where

$$
\begin{gathered}
S_{1}=\left\{v_{i, 1}: 1 \leq i \leq m\right\} \\
S_{2}=\left\{v_{i, j}: 1 \leq i \leq m, 2 \leq j \leq n-1\right\}
\end{gathered}
$$

and

$$
S_{3}=\left\{v_{i, n}: 1 \leq i \leq m\right\} .
$$

To obtain $D S\left(C_{m} \triangleright P_{n}\right)$ from $C_{m} \triangleright P_{n}$, we add three vertices $w_{1}, w_{2}$ and $w_{3}$ corresponding to $S_{1}, S_{2}$ and $S_{3}$ respectively. Thus we get $V\left(D S\left(C_{m} \triangleright P_{n}\right)\right)=$ $V\left(C_{m} \triangleright P_{n}\right) \cup\left\{w_{1}, w_{2}, w_{3}\right\}$. First we find the upper bound for $\chi_{s}\left(D S\left(C_{m} \triangleright P_{n}\right)\right)$.

The star chromatic number is defined as follows:

Case(i):

If $m=3 k, k \geq 1$ 
For $n \equiv 1(\bmod 3)$

$$
\begin{gathered}
\sigma\left(v_{i, 3 k-2}\right)=i(\bmod 3), \text { for } 1 \leq k \leq\left\lceil\frac{n}{3}\right\rceil \\
\sigma\left(v_{i, 3 k-1}\right)=i+1(\bmod 3), \text { for } 1 \leq k \leq\left\lfloor\frac{n}{3}\right\rfloor \\
\sigma\left(v_{i, 3 k}\right)=i+2(\bmod 3), \text { for } 1 \leq k \leq\left\lfloor\frac{n}{3}\right\rfloor
\end{gathered}
$$

and

$$
\sigma\left(w_{1}\right)=\sigma\left(w_{2}\right)=\sigma\left(w_{3}\right)=4 .
$$

For $n \equiv 2(\bmod 3)$

$$
\begin{gathered}
\sigma\left(v_{i, 3 k-2}\right)=i(\bmod 3), \text { for } 1 \leq k \leq\left\lceil\frac{n}{3}\right\rceil \\
\sigma\left(v_{i, 3 k-1}\right)=i+1(\bmod 3), \text { for } 1 \leq k \leq\left\lceil\frac{n}{3}\right\rceil \\
\sigma\left(v_{i, 3 k}\right)=i+2(\bmod 3), \text { for } 1 \leq k \leq\left\lfloor\frac{n}{3}\right\rfloor
\end{gathered}
$$

and

$$
\sigma\left(w_{1}\right)=\sigma\left(w_{2}\right)=\sigma\left(w_{3}\right)=4 .
$$

For $n \equiv 3(\bmod 3)$

$$
\begin{gathered}
\sigma\left(v_{i, 3 k-2}\right)=i(\bmod 3), \text { for } 1 \leq k \leq \frac{n}{3} \\
\sigma\left(v_{i, 3 k-1}\right)=i+1(\bmod 3) \text { for } 1 \leq k \leq \frac{n}{3} \\
\sigma\left(v_{i, 3 k}\right)=i+2(\bmod 3), \text { for } 1 \leq k \leq \frac{n}{3}
\end{gathered}
$$

and

$$
\sigma\left(w_{1}\right)=\sigma\left(w_{2}\right)=\sigma\left(w_{3}\right)=4 .
$$

Thus, $\chi_{s}\left(D S\left(C_{m} \triangleright P_{n}\right)\right)=4$ if $m=3 k, k \geq 1$.

Case(ii)(a): When $m=3 k+1, k \in N$.

We color the $3 k$ vertices of $C_{m}$ by $\sigma\left(v_{i, 1}\right)=i(\bmod 3)$ and we assign the remains of one uncolored vertex by 4 .

Also, we assign

$$
\sigma\left(v_{i, j}\right)=i+j-1(\bmod 3) .
$$


and

$$
\sigma\left(w_{1}\right)=\sigma\left(w_{2}\right)=\sigma\left(w_{3}\right)=5 .
$$

Case(ii)(b): When $m=3(k-1)+2, k \in N$, here $m=5$ is not included. That is $m=3(k-1)+5, k \geq 2$.

We color the $3(k-1)$ vertices of $C_{m}$ by 1,2 and 3 and for the remaining five vertices assign the color 4, 1, 2, 3, 4 .

Also, we assign

$$
\sigma\left(v_{i, j}\right)=i+j-1(\bmod 3)
$$

and

$$
\sigma\left(w_{1}\right)=\sigma\left(w_{2}\right)=\sigma\left(w_{3}\right)=5 .
$$

Thus, $\chi_{s}\left(D S\left(C_{m} \triangleright P_{n}\right)\right)=5$.

When $m=5$, then $\chi_{s}\left(D S\left(C_{m} \triangleright P_{n}\right)\right)=5$. Hence, the theorem is proved.

\section{REF EREN C E S}

1. M. O. Albertson, G. G. Chappell, H. A. Kiestead, A. Kündgen and R. Ramamurthi: Coloring with no 2-colored $P_{4}$ 's. Electron. J. Comb. 11 (2004), Paper \# R26.

2. R. Alfarisi and D. Darmaji: On the star parition dimension of comb product of cycle and complete graph. In: International Conference on Mathematics: Education, Theory and Application, IOP series Journal of physics: Conference series, 855 2017, 012005.

3. L. Barriére, F. Comellas, C. DAfió and M. A. Fiol: The hierarchical product of graphs. Discrete Appl. Math. 157 (2009), 39-48.

4. J. A. Bondy and U. S. R. MURTY: Graph theory with applications. MacMillan, London, 1976.

5. J. Clark and D. A. Holton: A first look at graph theory. World Scinetific, 1969.

6. T. F. COLEMAN and J. MoRÉ: Estimation of sparse Hessian matrices and graph coloring problems. Math. Program. 28(3) (1984), 243-270.

7. D. DARMAJi and R. Alfarisi: On the parition dimension of comb product of path and complete graph. In: International Conference on Mathematics: Pure, Applied and Computation, AIP Conf.Proc.1867, 020038-1-020038-7.

8. G. Fertin, A. Raspaud and B. Reed: On Star coloring of graphs. J. Graph theory. 47(3) (2004), 163-182.

9. B. Grünbaum: Acyclic colorings of planar graphs. Israel J. Math. 14 (1973), 390-408.

10. F. Harary: Graph theory. Narosa Publishing home, New Delhi 1969.

11. R. Ponraj and S. Somasundaram: On the degree splitting graph of a graph. Natl. Acad. Sci. Lett. 27 (7-8) (2004), 275-278.

12. E. Sampathkumar and H. B. Walikar: On splitting graph of a graph. Journal of Karnatak University Science, 25 \& 26 (Combined) (1980-81), 13-16. 
13. S. W. Saputro, N. Mardiana and I. A. Purwasih: The metric dimension of comb product graphs. In: Graph theory conference in honor of Egawa's 60th birthday, 2013, pp. 10-14.

14. M. Tavakoli, F. Rahbarnia and A. R. Ashrafi: Distribution of some graph invariants over hierarchical product of graphs. Appl. Math. Comput. 220 (2013), 405-413.

\author{
Ulagammal Subramanian \\ Department of Mathematics \\ University College of Engineering Nagercoil \\ (A Constituent College of Anna University, Chennai) \\ Konam, Nagercoil- 629004 \\ Tamil Nadu \\ India \\ ulagammal2877@gmail.com
}

Vernold Vivin Joseph

Department of Mathematics

University College of Engineering Nagercoil

(A Constituent College of Anna University, Chennai)

Konam, Nagercoil- 629004

Tamil Nadu

India

vernoldvivin@yahoo.in 